\title{
Serum free amino acid profiling in differential diagnosis of ovarian tumors - a case control study with review of the literature
}

Agnieszka Horala ( $\sim$ agnieszka0lemanska@gmail.com )

Uniwersytet Medyczny imienia Karola Marcinkowskiego w Poznaniu https://orcid.org/0000-00025390-9213

\section{Szymon Plewa}

Poznan University of Medical Sciences: Uniwersytet Medyczny imienia Karola Marcinkowskiego w

Poznaniu

\section{Pawel Derezinski}

Poznan University of Medical Sciences: Uniwersytet Medyczny imienia Karola Marcinkowskiego w Poznaniu

\section{Agnieszak Klupczynska}

Uniwersytet Medyczny im Karola Marcinkowskiego w Poznaniu: Uniwersytet Medyczny imienia Karola Marcinkowskiego w Poznaniu

\section{Jan Matysiak}

Poznan University of Medical Sciences: Uniwersytet Medyczny imienia Karola Marcinkowskiego w Poznaniu

\section{Ewa Nowak-Markwitz}

Poznan University of Medical Sciences: Uniwersytet Medyczny imienia Karola Marcinkowskiego w Poznaniu

\section{Zenon J Kokot}

Akademia Kaliska

\section{Research}

Keywords: Ovarian cancer, ovarian neoplasm, biomarker, amino acids, metabolomics, metabolic profiling

Posted Date: November 18th, 2020

DOl: https://doi.org/10.21203/rs.3.rs-109758/v1

License: (9) This work is licensed under a Creative Commons Attribution 4.0 International License. Read Full License 
1 Serum free amino acid profiling in differential diagnosis of ovarian tumors $-\mathbf{a}$ case control study with review of the literature

3

4 Agnieszka Horala ${ }^{*}$, Szymon Plewa $^{2}$, Pawel Derezinski², Agnieszka Klupczynska ${ }^{2}$, Jan 5 Matysiak $^{2}$, Ewa Nowak-Markwitz ${ }^{1}$, Zenon J. Kokot ${ }^{3}$

6

$7{ }^{1}$ Gynecologic Oncology Department, Poznan University of Medical Sciences, Polna 33 Street, 8 Poznan 60-535, Poland;

9

${ }^{2}$ Department of Inorganic and Analytical Chemistry, Poznan University of Medical Sciences, Grunwaldzka 6 Street, Poznan 60-780, Poland;

${ }^{3}$ Faculty of Health Sciences, Akademia Kaliska im. Prezydenta Stanisława Wojciechowskiego, 62-800 Kalisz, Poland; zkokot@ump.edu.pl (Z.J.K.)

* Corresponding author

Agnieszka Horala, PhD

Gynecologic Oncology Department

Poznan University of Medical Sciences,

Polna 33 Street, Poznan 60-535, Poland

email address: agnieszka0lemanska@gmail.com 


\section{Abstract}

\section{Background}

Due to lack of effective early diagnostic methods, ovarian cancer (OC) remains a disease with a very unfavorable prognosis and correct differentiation between benign and malignant ovarian tumors is often difficult. Metabolomic profiling has recently been widely used in the search for non-invasive cancer diagnostic methods. In this research the serum free amino acid profiles were investigated to identify potential novel biomarkers of $\mathrm{OC}$ and assess their performance in ovarian tumor differential diagnosis.

\section{Results}

Serum samples from patients diagnosed with ovarian tumors were divided based on the histopathological result: epithelial OC $(n=38)$, borderline ovarian tumors $(n=6)$ and benign ovarian tumors (BOTs) $(\mathrm{n}=62)$. Serum free amino acid profiles were evaluated using aTRAQ methodology based on high-performance liquid chromatography electrospray ionization tandem mass spectrometry. In the performed analyses, the levels of eleven amino acids significantly differed between OC + borderline and BOTs. The highest AUC (0.787) was obtained for histidine. Cystine and histidine were identified as best single markers for early stage OC/BOT and type I OC. For advanced stage OC, seven amino acids differed significantly between the groups and citrulline obtained the best AUC of 0.807. Between type II OC and BOTs eight amino acids differed significantly and the highest AUC of 0.798 was achieved by histidine and citrulline (AUC of 0.778).

\section{Conclusions}


46 Serum free amino acid profiles are significantly altered in OC patients as compared to those

47 with BOTs. Histidine emerges as a potential new biomarker in differential diagnosis of ovarian tumors. Adding histidine to a multimarker panel together with CA125 and HE4 improved the differential diagnosis between OC and BOTs.

50

51 Keywords

52 Ovarian cancer, ovarian neoplasm, biomarker, amino acids, metabolomics, metabolic profiling

53

54 


\section{Background}

The research for elaborating efficient ovarian cancer (OC) diagnostic tools has been ongoing for decades. Until now, no screening method is available and the disease has a very unfavorable prognosis, mainly due to the fact that over $70 \%$ of the patients are diagnosed in late stages, i.e. stage III and IV according to the International Federation of Gynaecology and Obstetrics (FIGO). Early and specific diagnosis is essential to improve the treatment outcome, as five-year survival rates for FIGO stage I reach $90 \%$ as compared to about $30 \%$ for advanced disease (FIGO stage III-IV) (1).

One of the challenges in OC diagnosis is the correct differentiation of ovarian tumors noticed on routine transvaginal ultrasound examination. So far, histological examination of the resected tissue still remains the golden standard. This approach results in unnecessary surgical procedures that, if a reliable non-invasive diagnostic method existed, could be avoided as over $90 \%$ of ovarian masses detected in pre-menopausal women and up to $60 \%$ of those in postmenopausal women are benign (2). Moreover, correct pre-operative diagnosis of OC enables adequate referral of the patient to specialized gynecologic oncology centers where evaluation by an interdisciplinary tumor board and optimal debulking surgery is possible. Treating women with OC in specialized centers is crucial to ensure proper management and was proved to significantly improve the prognosis (3).

Some clinical multivariate diagnostic models used in ovarian tumor differential diagnosis were reported to be quite efficient, for example ADNEX model, based on ultrasound features and clinical data, was reported to reach an area under the receiver operating characteristic (AUC of ROC) curves as high as 0.954 (4). However, despite the excellent performance, its clinical 
application is highly limited due to the need for highly-trained medical staff and modern equipment to perform high-quality ultrasound assessment of ovarian tumors and record the required features for the model. For this reason, biomarker research is more likely to provide accessible and ready-to-use diagnostic methods.

Metabolomic profiling has recently become a very promising target in the search for noninvasive cancer diagnostic methods. Metabolome is defined as a complete set of smallmolecules within a biological sample. Therefore, it is a direct reflection of the current processes in the organism and is altered by pathological conditions such as carcinogenesis. Free amino acids profiling is one of the metabolomic approaches because many proteinogenic (used to build proteins) and non-proteinogenic amino acids play critical roles within the body, e.g. act as neurotransmitters (glutamic acid), form connective tissue (hydroxyproline), are used to synthesize porphyrins in erythrocytes (glycine) or help in lipid transport (carnitine). Several studies revealed an alteration of plasma/serum free amino acid (PFAA/SFAA) profile in patients with cancer, e.g. lung, gastric, colorectal, renal, breast or prostate (5-7).

The purpose of this study was to investigate the differences in the SFAA profile in patients with OC and BOT and assess the diagnostic utility of relevant amino acids in pre-surgical differential diagnosis of ovarian tumors. To the best of our knowledge, this is the first study in ovarian cancer that analyzes such a wide spectrum of the SFAA profile in differential diagnosis of ovarian tumors.

\section{Results}


Out of 42 analyzed amino acids, 33 were included in the final analysis due to the fact that the concentrations of 9 amino acids did not exceed the limit of quantitation (LOQ).

There were no statistically significant differences in body mass index (BMI) between the studied groups. As some conditions, such as hepatitis, cirrhosis, diabetes or malnutrition (defined as weight loss $>10 \%$ in the past 3 months), were reported to alter PFAA profiles (8), they were among the exclusion criteria. Cachectic patients were excluded from the analysis therefore malnutrition was not expected to influence the results. Significant differences in age as well as menopausal status were observed among the groups, as expected in a real-life population taking into account the disease incidence. Detailed study group characteristics are presented in Table 1.

All borderline tumors and OC patients underwent complete surgical staging according to the classification by FIGO. Approximately one-third of OC patients had early stage disease (FIGO I-II) (Table 2).

Table 1. Study group characteristics.

\begin{tabular}{|l|l|l|l|l|l|}
\hline & \multicolumn{3}{|c|}{ Ovarian cancer } & $\begin{array}{l}\text { Borderline } \\
\text { tumors }\end{array}$ & \multicolumn{1}{c|}{ BOTs } \\
\hline & Total & Type I & Type II & & \\
\hline $\begin{array}{l}\text { Number of samples } \\
(\%)\end{array}$ & $38(24.4)$ & $7(4.5)$ & $31(19.9)$ & $6(3.9)$ & $62(39.7)$ \\
\hline $\begin{array}{l}\text { Age (years) median } \\
\text { (range) }\end{array}$ & $60(32-78)$ & $54(32-70)$ & $63(36-78)$ & $48(37-52)$ & $40.5(17-72)$ \\
\hline $\begin{array}{l}\text { BMI median } \\
\text { (range) }\end{array}$ & $\begin{array}{l}25.1 \\
(18.6-38.4)\end{array}$ & $\begin{array}{l}26.0(18.6- \\
36.9)\end{array}$ & $\begin{array}{l}25.0(20.7- \\
38.4)\end{array}$ & $\begin{array}{l}27.3 \\
(17.3-31.6)\end{array}$ & $\begin{array}{l}24.3 \\
(17.9-39.9)\end{array}$ \\
\hline
\end{tabular}




\begin{tabular}{|l|l|l|l|l|l|}
\hline $\begin{array}{l}\text { \% of } \\
\text { postmenopausal }\end{array}$ & 79 & 57 & 84 & 33 & 26 \\
\hline FIGO stage, $\boldsymbol{n}(\boldsymbol{\%})$ & & & & & \\
\hline I & $10(26.3)$ & $4(57.1)$ & $6(19.4)$ & $6(100)$ & N/A \\
\hline II & $2(5.3)$ & 0 & $2(6.5)$ & 0 & N/A \\
\hline III & $25(65.8)$ & $3(43.9)$ & $22(71.0)$ & 0 & N/A \\
\hline IV & $1(2.6)$ & 0 & $1(3.2)$ & 0 & N/A \\
\hline $\begin{array}{l}\text { Histopathological } \\
\text { type, } \boldsymbol{n}(\boldsymbol{\%})\end{array}$ & & & & & \\
\hline Serous & & & & & \\
\hline Endometrioid & $4(10.5)$ & 0 & $4(10.5)$ & $1(16.7)$ & $18(29.0)$ \\
\hline Mucinous & $1(2.6)$ & $1(2.6)$ & 0 & $1(16.7)$ & $2(3.2)$ \\
\hline Clear cell & $3(7.9)$ & $3(7.9)$ & 0 & 0 & N/A \\
\hline Undifferentiated & $10(26.3)$ & 0 & $10(26.3)$ & 0 & N/A \\
\hline Non identified & $4(10.5)$ & 0 & $4(10.5)$ & 0 & N/A \\
\hline Teratoma & N/A & N/A & N/A & N/A & $11(17.7)$ \\
\hline Other & N/A & N/A & N/A & N/A & $17(27.4)$ \\
\hline
\end{tabular}

The results of the statistical analyses are presented in Tables 2-3 and S1.

A. Usefulness of amino acid profiling in differential diagnosis of ovarian tumors (OC

\section{vs BOTs) and (OC+borderline tumors vs BOTs)}

In the performed analyses, the levels of ten amino acids significantly differed between OC and BOT (elevated in OC: Aad, Cys, Ile, Leu; decreased in OC: Asn, Cit, Gln, His, Thr, Trp). When borderline tumors were added to the OC group, the level of one additional amino acid (Phe) was significantly increased in the OC/borderline tumors group. The highest AUC in both analyses ( 0.820 and 0.787 , respectively) was obtained by histidine which level was significantly reduced in the OC/borderline tumors patients. However, none of the analyzed amino acids obtained an AUC superior to those of CA125 and HE4 (Table 2). 
In order to further evaluate the performance of histidine, multivariate models based on two variables (CA125+HE4) and three variables (CA125+HE4+histidine) were created. The analysis revealed that adding histidine to a multi-marker panel improved the diagnostic performance of the test in both analyses (Table 3).

\section{B. Usefulness of amino acid profiling in detecting early stages of ovarian cancer} (FIGO I-II (incl. borderline tumors) vs benign and FIGO III-IV vs benign)

In order to assess the ability of the analyzed markers to detect early stage disease, two subgroups of OC patients (FIGO stage I-II and FIGO stage III-IV) were independently compared against benign ovarian tumor group. This analysis included borderline tumors and all of them were staged FIGO I. In the first analysis, the levels of two amino acids (Cys and His) differed significantly between early stage OC and BOT, whereas in advanced stage OC the levels of seven amino acids were significantly different than in BOT (Aad, Cut, Gln, His, Ile, Thr, Trp). These results confirm that the SFAA profiles become increasingly altered when the malignancy progresses. The highest AUC in early stage OC was achieved by histidine (0.786) and in advanced stage OC by citrulline (0.807), although histidine also obtained a high AUC of 0.788 . Again, none of the analyzed amino acids obtained an AUC superior to those of CA125 and HE4 (Table 2). As expected, the discriminatory ability of CA125 and HE4 was higher in advanced stage OC.

\section{The addition of histidine improved the diagnostic accuracy of a multivariate model for early} stage disease (FIGO I-II vs BOT) and the addition of citrulline improved the diagnostic 
accuracy of a multivariate model for advanced stage disease (FIGO III-IV vs BOT), however the raise in the AUC value was negligible (Table 3).

\section{Usefulness of amino acid profiling in distinguishing ovarian cancer types (type I} $\underline{\text { OC vs type II OC, type I OC vs benign, type II OC vs benign) }}$

Having in mind the heterogeneity of OC, this set of analyses was performed to investigate if two clinically and molecularly distinct subtypes of OC (according to Kurman et al. (9)) could possibly have their own specific markers. Borderline tumors were included in this analysis as type I OC. Interestingly, only citrulline was expressed differently in type I OC than in type II OC (AUC of 0.730). The levels of histidine, which obtained the highest AUC in almost all other analyses, did not differ significantly between type I and type II OC which may be suggestive of its universal role in OC diagnosis. In the comparison between type I OC and BOT two amino acids were expressed differently (Cys and His) and these were the same ones that were useful in detecting early stage OC. The fact that type I OC is generally characterized by less aggressive clinical course and thus is more likely to be diagnosed in early stages, corresponds well with these results. When comparing type II OC with BOT, eight amino acids significantly differentiated the groups (Aad, Cit, Gln, His, Ile, Leu, Phe, Trp). The highest discriminatory ability was again achieved by histidine (AUC of 0.798 ), closely followed by citrulline (AUC of 0.778) which may suggest citrulline as a type II OC marker (particularly as it did not reach statistical significance in type I OC vs BOT analysis). Additionally, cystine could be distinguished as a potential type I OC marker as it was one of the few amino acids that did reach statistical significance in the type I vs BOT analysis. 
Both, CA125 and HE4, obtained high AUC in all analyses, however their discriminatory ability was significantly higher for type II OC (AUC of 0.965 and 0.972 , respectively) than for type I OC (AUC of 0.810 and 0.828 , respectively).

In multivariate model analyses the addition of histidine to a two-marker panel consisting of CA125 and HE4 only slightly raised respective AUC values (Table 3).

Table 2. Serum free amino acids and OC markers (CA125 and HE4) showing significant pvalues $(\mathrm{p}<0.05)$ and corresponding AUC of ROC in differential diagnosis between the analyzed groups. The highest obtained AUC in each group are bolded.

[Table 2 provided in a separate file.]

Table 3. Areas under the receiver operating characteristic (AUC of ROC) curves for multivariate models comparing their diagnostic utility in differential diagnosis between the analyzed groups.

\begin{tabular}{|c|l|c|c|c|}
\hline $\begin{array}{c}\text { Result } \\
\text { S } \\
\text { sectio } \\
\text { n }\end{array}$ & \multicolumn{1}{|c|}{ Analyzed groups } & $\begin{array}{c}\text { 2-marker } \\
\text { model } \\
\text { AUC (CI } \\
\text { 95\%) }\end{array}$ & \multicolumn{2}{|c|}{$\begin{array}{c}\text { 3-marker model } \\
\text { AUC (CI 95\%) }\end{array}$} \\
\hline & & CA125+HE4 & $\begin{array}{c}\text { CA125+HE4 } \\
\text { +histidine }\end{array}$ & $\begin{array}{c}\text { CA125+HE4+ } \\
\text { citrulline }\end{array}$ \\
\hline A & ovarian cancer (OC) vs BOT & 0.988 & 0.995 & X \\
& & $(0.965-)$ & $(0.981)$ & \\
\hline A & OC+borderline ovarian tumors & 0.938 & 0.955 & X \\
& vs BOT & $(0.863-)$ & $(0.893-)$ & $\mathrm{X}$ \\
\hline B & FIGO stage I-II OC vs BOT & 0.839 & 0.873 & $(0.710-0.987)$ \\
& & & & \\
\hline
\end{tabular}




\begin{tabular}{|c|l|c|c|c|}
\hline $\mathbf{B}$ & FIGO stage III-IV OC vs BOT & $\begin{array}{c}0.996 \\
(0.978-)\end{array}$ & $\mathrm{x}$ & $\begin{array}{c}0.999 \\
(0.996-)\end{array}$ \\
\hline $\mathbf{C}$ & Type I OC vs BOT & 0.802 & 0.822 & $\mathrm{x}$ \\
& & $(0.523-)$ & $(0.575-0.998)$ & \\
\hline $\mathbf{C}$ & Type II OC vs BOT & 0.988 & 0.993 & $\mathrm{x}$ \\
& & $(0.961-)$ & $(0.974-))$ & \\
\hline
\end{tabular}

\section{Discussion}

For many years, inefficient attempts have been made to find a method for early detection of

OC. As ovaries are relatively inaccessible organs and as most ovarian masses are benign, ideally the diagnosis of OC should be obtained from an accessible body fluid, such as blood, urine or saliva. Moreover, due to rarity of this cancer, potential markers should have high sensitivity and specificity to ensure cost-effectiveness. In recent years, thanks to technological advances, metabolomics has emerged as a promising method of search for new OC biomarkers.

A strength of our research is the adoption of state-of-the-art aTRAQ methodology based on high-performance liquid chromatography electrospray ionization tandem mass spectrometry (HPLC-ESI-MS/MS) that decreased the required sample volume, enhanced specificity and significantly reduced time of chromatographic separation compared to conventional amino acids quantitation basing on ion exchange chromatography (IEC) followed by post-column ninhydrin derivatization and UV detection. Moreover, quantitation based on labeled internal standard for each analyte ensured high accuracy and reliability of the metabolomic data (10). Owing to the small sample volume required $(40 \mu \mathrm{L})$ and short time of analysis per sample needed (18 minutes), this high-throughput method emerges as promising approach in the contemporary biomarker research. 
Amino acids are the key components of peptides and proteins and are essential for cancer growth. Changes in their availability have profound effects on many aspects of cellular functions such as cell signaling, gene expression and transportation of amino acids themselves (11). PFAA/SFAA profiles reflect the physiological or pathological conditions of an organism. Alterations in the PFAA/SFAA profiles can be attributed to malnutrition in cancer patients (therefore most studies, including ours, exclude cachectic patients from the analysis) and to the hypermetabolic changes such as increased lipolysis, fatty acid oxidation and whole-body protein catabolism (12). Several studies confirmed that PFAA/SFAA profile is significantly altered in cancer patients, e.g. lung, gastric, colorectal, breast, renal, prostate and endometrial cancers $(5-7,13,14)$. However, studies on PFAA/SFAA profiling in ovarian cancer are very scarce.

Our results identified histidine as the most effective OC marker in almost all analyzed subgroups. What is especially valuable, its performance did not drop for detection of earlystage cancer (AUC of 0.786 and 0.788 for early and late-stage OC, respectively). Moreover, it obtained similar results in the comparison between type I or type II OC with BOT and did not differ significantly between the two OC types. Therefore, it could be considered as a universal OC biomarker and should undergo further research. Histidine was closely followed by tryptophan which obtained high AUC values, especially in advanced stage and high-grade (type II) OC. The depletion of those two amino acids in OC patients are in line with the recent studies - see Table 4.

The results of our study also point at citrulline that could be considered as a type II OC marker (particularly as it did not reach statistical significance in type I OC vs BOT analysis). 
Additionally, cystine could be distinguished as a potential type I OC marker as it was one the few amino acids that did reach statistical significance in the type II vs BOT analysis. However, the performance of these two amino acids may not be sufficient for clinical use.

Table 4. Overview of metabolomic studies on OC and their results: concentrations of several amino acids were significantly different in OC as compared to BOT and/or healthy controls.

\section{[Table 4 provided in a separate file.]}

Our findings correspond with the results of a paper on high-grade serous OC (equivalent to type II OC in our study) using targeted metabolomics which reports a decreased serum concentrations of five amino acids (histidine, lysine, threonine, tryptophan, citrulline) compared with healthy controls that also correlated with shorter overall survival of cancer patients (18). The levels of four of these amino acids (histidine, threonine, tryptophan, citrulline) were decreased in OC patients in our analysis and three (histidine, tryptophan, citrulline) were decreased in type II OC, although it has to be noted that the comparison was between OC and BOT (not healthy controls). Other important findings of the above-mentioned study are that the levels of amino acids identified as significant were similar in serum, ascites fluid and tumor tissue and that they were positively correlated with the tumor load (i.e. recovered to concentrations typical for healthy patients after initiation of anti-cancer treatment). The authors conclude that this suggests that the depletion of certain amino acids in serum is a direct effect of tumor metabolism (18).

Other studies that analysed serum samples identified altered levels of several amino acids in OC patients. These results are summarized in Table 4. There are a lot of discrepancies in the 
amino acids identified as differential and some studies even reveal an opposite trend of a specific metabolite (e.g. alanine, threonine). This might be due to the adoption of different mass spectrometry-based analytic methods to identify those metabolites and different study design, especially control groups. Moreover, all cited studies were based on global rather than targeted metabolomic profiling techniques in which amino acids were only a small proportion of the investigated substances whereas our study is unique in that it focused purely on SFAA profile. Notwithstanding different study methods, the results are coherent for histidine and tryptophan which suggests that their levels are strongly affected by OC development .

A study by Hilvo et al. (16) additionally compared the results obtained from serum samples with matching tumor tissue samples and confirmed a linear correlation of diagnostically relevant biomarkers between serum and tumor tissue. These findings support the hypothesis that relevant metabolites originate from the tumor rather than depend on other metabolic processes in the body.

The study by Miyagi et al. (21) investigated the ability of amino acid profile-based index to discriminate OC/borderline tumors from BOT in comparison to CA125 and revealed their equivalent performance (AUC of ROC of 0.77 for both tests) and better performance of the amino acid test for discrimination between malignant/borderline malignant lesions and endometriotic cysts (0.75 vs.0.59). However, the study does not reveal on which amino acids the index was based and only mentions the detection of increased concentrations of isoleucine and proline and decreased of histidine and tryptophan in OC reported in earlier phase of the research (those results were published in Japanese). A decreased level of tryptophan was also reported in a study based on metabolomic profiling that analyzed a broad spectrum of 535 metabolites (22) - this amino acid was selected as one of the best six biomarkers and was 
suspected to participate in cancer progression $(5,22)$. Another study based on mass spectrometry performed metabolic profiling of 448 plasma samples comparing patients with OC with BOT or uterine fibromas patients and identified fifty-three differential metabolites, among them decreased levels of several amino acids: tryptophan, histidine, phenylalanine and lysine. Diagnostic models composed of newly selected metabolites were superior in ovarian tumor differential diagnosis (OC vs BOT) to CA125 (AUC 0.910 vs 0.848 ) (20) In our study we also observed an increase in the level of isoleucine (but not proline) and a decrease in histidine and tryptophan in OC patients.

It is not clear, however, to which extent the studies based on plasma analysis can be compared with our research in which serum samples were collected. Serum is the liquid fraction of whole blood obtained after the blood is allowed to clot and centrifuged. Plasma is obtained when whole blood is collected in tubes treated with an anticoagulant and then centrifuged to remove blood cells. What may seem surprising, a study comparing amino acid profiles in both types of blood samples revealed remarkable differences in the PFAA and SFAA profiles (7). In general, the amino acid concentrations were averagely $40 \%$ lower in plasma than in serum, although the level of variation and the direction of changes varied for each individual amino acid. Nevertheless, significant differences were observed in both profiles (SFAA and PFAA) between cancer patients (clear cell renal cancer) and healthy controls and in serum a decreased level of histidine - the same as in our study - was identified as the most effective cancer marker (7).

Apart from the SFAA profile, in our research two clinically used OC biomarkers, CA125 and HE4, were additionally analyzed. Their generally high performance in differential diagnosis of ovarian tumors was also confirmed by our analyses. As expected, their diagnostic accuracy was lower in detecting early-stage and type I OC. Although all of the analyzed amino acids failed 
to reach a higher AUC than CA125 and HE4, the diagnostic performance of histidine was not subject to OC stage and type. Moreover, the addition of histidine improved the diagnostic performance of all presented multivariate models based on CA125 and HE4.

\section{Most of the amino acids identified in our research as statistically significant were proved to be} involved in metabolic pathways altered during cancer growth and progression. Tryptophan depletion triggers apoptosis of effector $\mathrm{T}$ cells contributing to the suppression of antitumor immune responses (23). Considerable evidence indicates that histamine, a derivative of amino acid histidine, may be a crucial mediator in cancer growth and progression by regulating processes such as angiogenesis, cell invasion, migration, differentiation, apoptosis and modulation of immune responses (24). Histidine decarboxylase that converts histidine to histamine was found to be overexpressed in several cancers, including OC tissue (25). Glutamine is used by tumors for nucleotide biosynthesis whereas glutamate, its derivative, serves as a donor of nitrogen for the production of other amino acids. Glutaminase, an enzyme which converts glutamine to glutamate, was found to be frequently upregulated in cancer cells $(23)$.

Among the limitations of this study are the number of patients and the fact that they were all from a single institute. On the other hand, this ensured the consistency in gathering and processing the samples. The distribution of histological types of OC consisted of serous (42\%), endometrioid (11\%), clear cell (8\%), mucinous (3\%) and undifferentiated carcinomas (26\%), and the frequency of the last type is much higher than reported in other European countries. This is probably due to an individual bias of the pathology department and some of these 
cancers could probably have been classified as high-grade serous. In 4 cases (10\%) the type was not identified because the patients were qualified to neoadjuvant chemotherapy and the diagnosis was obtained after a paracentesis of ascites. The study also excluded other than epithelial OC (i.e. germ-cell and stromal cancers). However, taking into account their extremely low incidence (less than $2 \%$ of cancers) this factor has very limited clinical impact. Another potential weakness of the presented research is the possibility of relation between behavioral and/or dietary patterns of the patients and alterations in the amino acid profiles (26). To reduce this potential bias, cachectic patients were excluded from the analysis and the blood samples were collected after overnight fasting.

The number of patients in the subgroup analyses (early vs late stage; type I vs type II) was especially limited therefore much larger cohorts are needed to verify the utility of the amino acids indicated in these subgroups as relevant. Nevertheless, since only diagnosis at an early, asymptomatic stage is likely to have a significantly impact on the clinical outcomes of OC patients, the subgroup analyses provide important input. The presented study examined the role of SFAA profiles in differential diagnosis of ovarian tumors and assessed the performance of several multimarker models for pre-surgical evaluation of ovarian masses. A possible direction of future research could be the assessment of SFAA profiles in OC screening, before the actual ovarian tumor is observed in ultrasound examination.

\section{Conclusions}

This is, to the best of our knowledge, the first study analyzing SFAA profiles in differential diagnosis of ovarian tumors. SFAA profiles were proved to be significantly altered in OC 
cancer patients as compared to patients with BOT. The results of this study pointed at histidine that emerges as a possible new OC biomarker. Adding histidine to a multimarker panel together with CA125 and HE4 may improve the differential diagnosis of ovarian tumors. The results of subgroup analyses indicate that citrulline may be a potential advanced stage and/or type II OC biomarker and cystine a potential early stage and/or type I OC biomarker. The findings of this research may give grounds to the development of more effective novel OC diagnostic methods that are needed to ensure proper qualification for surgical treatment and to avoid overtreatment of women with ovarian tumors. Further studies are needed to determine the role of SFAA alterations in OC and select valuable biomarkers for practical use in the future.

\section{Methods}

The purpose of this case control study was to compare the SFAA profiles between ovarian cancer patients and patients with benign ovarian tumours, select differentiating amino acids between the groups and assess their performance in differential diagnosis of ovarian tumours . Blood samples were collected from 122 patients diagnosed with ovarian tumors and qualified for surgical treatment in Gynecologic Oncology Department between August 2014 and December 2015. A written consent to participate in the study was obtained from all patients prior to sample collection. All blood samples $(7.5 \mathrm{~mL})$ were collected using the same type of vials on the day of the operation after overnight fasting in order to reduce the diet induced differences. After collection, the samples were incubated for 30 minutes in room temperature for clotting, then centrifuged for 15 minutes at $4000 \mathrm{rpm}$ at $4^{\circ} \mathrm{C}$. Serum was isolated and stored at $-80^{\circ} \mathrm{C}$ until analysis. The exclusion criteria were: any other malignancy currently or in anamnesis, ovarian malignancy other than epithelial OC, previous OC treatment, chronic liver diseases, diabetes, chronic renal failure and cachexia and were met by 16 patients. Samples 
from 106 patients were included in the final analysis and divided based on the histopathological result: OC $(n=38)$, borderline ovarian tumors $(n=6)$ and BOT $(n=62)$. In addition, OC group was divided into type I OC ( $\mathrm{n}=13$; borderline tumors were included in this group) and type II OC ( $\mathrm{n}=31)$ according to the clinicopathological classification proposed by Kurman (9).

As our study focused on differential diagnosis of ovarian tumors, borderline tumors were included in all analyses to better reflect the real population and avoid study selection bias. Taking into account the increasing evidence that low-grade serous carcinomas (type I OC) develop from borderline tumors and that the pathways and genes involved in their pathogenesis are distinct from those of high-grade serous carcinomas (type II OC) $(9,27)$, borderline tumors were added to type I OC group in for statistical analyses.

\section{Amino acid profiling}

In order to measure the SFAA concentrations, the aTRAQ kit for Amino Acid Analysis of Physiological Fluids (Sciex, Framingham, MA, USA) was used. The kit included all reagents to perform complete amino acids assay. LC-MS grade methanol was supplied by J.T. Baker (Griesheim, Germany). Deionized water $(18.2 \mathrm{M} \Omega / \mathrm{cm})$ was obtained by us using Direct-Q Merck Millipore (Darmstadt, Germany) water purifying system.

A panel composed of 42 amino acids and biogenic amines (Table 5) was quantitatively measured in all 106 samples. Out of those, 20 amino acids are encoded in the standard genetic code and are proteinogenic which means they are used to biosynthesize proteins during translation; all of these amino acids were included into the final analysis.

Determination of SFAA was carried out on the 4000 QTRAP mass spectrometer (Sciex, Framingham, MA, USA) coupled with 1260 Infinity high performance liquid chromatograph (HPLC) (Agilent Technologies, Santa Clara, CA, USA) system. The analyses were performed 
according to Sciex protocol using the aTRAQ methodology. The method was validated and described in detail by us earlier (28). The compounds of interest were separated on Sciex C18 $(5 \mu \mathrm{m}, 4.6 \mathrm{~mm} \times 150 \mathrm{~mm})$ column maintained at $50^{\circ} \mathrm{C}$. The mobile phase consisted of solvent A - water, and solvent B - methanol, both with addition of $0.1 \%$ formic acid and $0.01 \%$ heptafluorobutyric acid. The gradient elution was the following: 0 to $6 \mathrm{~min}-$ linear from 2 to $40 \%$ mobile phase $\mathrm{B} ; 6$ to $10 \mathrm{~min}-40 \% \mathrm{~B} ; 10$ to $11 \mathrm{~min}$ - linear from $40 \%$ to $90 \% \mathrm{~B} ; 12$ to 13 min - linear from 90 to $2 \% \mathrm{~B}$ and 13 to $18 \mathrm{~min}-2 \% \mathrm{~B}$, the flow rate was maintained at 0.8 $\mathrm{mL} / \mathrm{min}$. The injection volume was set at $2 \mu \mathrm{L}$. The HPLC system was coupled with mass spectrometer equipped with electrospray ionization interface (ESI ion source). The analyses were performed in a positive ionization mode. The mass spectrometer was operating in highly selective scheduled Multiple Reaction Monitoring (sMRM) mode. Data acquisition and processing were carried out under control of Analyst 1.5.2 software (Sciex).

CA125 and HE4 serum concentrations were quantitatively measured by electrochemiluminescence immunoassay (ECLIA) on Roche Cobas System (Roche Diagnostics, Indianapolis, IN, USA) in the Central Hospital Laboratory according to the manufacturer's instructions. The standard cut-off values are $35 \mathrm{U} / \mathrm{mL}$ for CA125 and 140 pmol/L for HE4, however for the purpose of this study optimal cut-off levels were identified.

Table 5. List of analyzed amino acids and biogenic amines in serum.

\begin{tabular}{|c|c|}
\hline Full name & Abbreviation \\
\hline 1-Methyl-L-histidine & 1MHis \\
\hline 3-Methyl-L-histidine & $3 \mathrm{MHis}$ \\
\hline L- $\alpha$ - Aminoadipic acid & $\mathrm{Aad}$ \\
\hline L- $\alpha$-Amino-n-butyric acid & $\mathrm{Abu}$ \\
\hline L-Alanine & Ala* \\
\hline L-Anserine & Ans** \\
\hline L-Arginine & Arg* \\
\hline Argininosuccinic acid & Asa** \\
\hline
\end{tabular}




\begin{tabular}{|c|c|}
\hline L-Asparagine & Asn* \\
\hline L-Aspartic acid & Asp* \\
\hline D, L- $\beta$-Aminoisobutyric acid & bAib \\
\hline$\beta$-Alanine & bAla \\
\hline L-Carnosine & Car** \\
\hline L-Citrulline & Cit \\
\hline Cystathionine & Cth** \\
\hline L-Cystine & Cys* \\
\hline Ethanolamine & EtN \\
\hline$\gamma$-Amino-n-butyric acid & $\mathrm{GABA}^{* *}$ \\
\hline L-Glutamine & $\mathrm{G} \ln *$ \\
\hline L-Glutamic acid & Glu* \\
\hline Glycine & Gly* \\
\hline L-Homocitrulline & $\mathrm{Hci}^{* *}$ \\
\hline L-Homocystine & Hcy** \\
\hline L-Histidine & His* \\
\hline$\delta$-Hydroxylysine & $\mathrm{Hyl}^{* *}$ \\
\hline Hydroxy-L-proline & Hyp \\
\hline L-Isoleucine & Ile* \\
\hline L-Leucine & Leu* \\
\hline L-Lysine & Lys* \\
\hline L-Methionine & Met* \\
\hline L-Ornithine & Orn \\
\hline O-Phosphoethanolamine & PEtN \\
\hline L-Phenylanalanine & Phe* \\
\hline L-Proline & Pro* \\
\hline O-Phospho-L-serine & PSer** \\
\hline Sarcosine & Sar \\
\hline L-Serine & Ser* \\
\hline Taurine & Tau \\
\hline L-Threonine & Thr* \\
\hline L-Tryptophan & Trp* \\
\hline L-Tyrosine & Tyr* \\
\hline L-Valine & $\mathrm{Val}^{*}$ \\
\hline
\end{tabular}

\section{Data analysis}

435 The statistical assessment was carried out using STATISTICA 12.5 (StatSoft Inc., Tulsa, OK, USA) software and MetaboAnalyst web server (29). Firstly, the normality of distribution of all data sets was tested using the Shapiro-Wilk test. In case of non-normally distributed variables 
the Mann-Whitney U test was applied to evaluate the differences in SFAA between the OC

439 group and the benign ovarian tumor group. Conversely, when the data was normally distributed,

440 Levene's test was used to evaluate the equality of variances. When variances were equal, the t-

441 test was applied for further statistical assessment, otherwise Welch t-test was used. In all

442 statistical tests, p-value <0.05 was regarded as significant. In the second step, the univariate

443 ROC curves were created for each of the analyzed amino acid. Metabolites characterized by the

444 highest AUC in univariate ROC were selected to perform multivariate ROC curve analyses.

445 For this purpose, data normalization by sum, logarithm transformation and auto scaling were

446 carried out. Obtained AUC of ROC curves were compared to assess the discriminatory ability

447 of the models.

448

$449 \quad$ List of abbreviations

450 OC - ovarian cancer

451 CA125 - cancer antigen 125

452 HE4 - human epididymis protein 4

453 ROC - receiver operating characteristic

454 AUC - area under curve

455 BOT - benign ovarian tumor

456 PFAA - plasma free amino acids

457 SFAA - serum free amino acids

458 LOQ - limit of quantitation

459 BMI - Body Mass Index 
Declarations

\section{Ethics approval and consent to participate}

465 The study was conducted in accordance with the Declaration of Helsinki and the protocol was approved by the local Bioethical Commission of Poznan University of Medical Sciences, Poland (Decision No. 165/16). A written consent for inclusion was obtained from all participants prior to sample collection.

\section{Consent for publication}

470 Not applicable.

\section{Availability of data and materials}

472 The datasets used and analysed in the study are available from the corresponding author on 473 request.

\section{Competing interests}

475 The authors declare that they have no competing interests.

\section{$476 \quad$ Funding}

477 The project received support from the Polish National Science Centre (grant number:

478 2014/15/B/NZ7/00964). The funders did not participate in the study design, data collection 479 and analysis, decision to publish and manuscript preparation.

\section{Authors' contributions}

481 AH coordinated the study. AH, SP, AK, PD, JM, ENM and ZJK designed research. AH and 482 ENM contributed important samples. SP, AK and PD performed research. SP and AH collected 
data. SP and AH analyzed data. AH and SP wrote the manuscript. AK, PD, JM, ENM and ZJK critically revised the manuscript. All authors have read and approved the submitted manuscript.

\section{Acknowledgements}

Not applicable.

1. Paik ES, Lee Y-Y, Lee E-J, Choi CH, Kim T-J, Lee J-W, et al. Survival analysis of revised 2013 FIGO staging classification of epithelial ovarian cancer and comparison with previous FIGO staging classification. Obstet Gynecol Sci. Korean Society of Obstetrics and Gynecology; 2015 Mar;58(2):124-34.

2. Enakpene CA, Omigbodun AO, Goecke TW, Odukogbe A-T, Beckmann MW. Preoperative evaluation and triage of women with suspicious adnexal masses using risk of malignancy index. J Obstet Gynaecol Res. 2009 Feb;35(1):131-8.

3. Vernooij F, Heintz P, Witteveen E, van der Graaf Y. The outcomes of ovarian cancer treatment are better when provided by gynecologic oncologists and in specialized hospitals: A systematic review. Gynecol Oncol. 2007 Jun;105(3):801-12.

4. Van Calster B, Van Hoorde K, Valentin L, Testa AC, Fischerova D, Van Holsbeke C, et al. Evaluating the risk of ovarian cancer before surgery using the ADNEX model to differentiate between benign, borderline, early and advanced stage invasive, and secondary metastatic tumours: prospective multicentre diagnostic study. BMJ. $2014 ; 349$.

5. Miyagi Y, Higashiyama M, Gochi A, Akaike M, Ishikawa T, Miura T, et al. Plasma free amino acid profiling of five types of cancer patients and its application for early detection. PLoS One. Public Library of Science; 2011;6(9):e24143. 
506 6. Lai H-S, Lee J-C, Lee P-H, Wang S-T, Chen W-J. Plasma free amino acid profile in cancer patients. Semin Cancer Biol. 2005;15(4):267-76.

7. Lee H-O, Uzzo RG, Kister D, Kruger WD. Combination of serum histidine and plasma tryptophan as a potential biomarker to detect clear cell renal cell carcinoma. J Transl Med. BioMed Central; 2017 Apr 6;15(1):72.

8. Kimura T, Noguchi Y, Shikata N, Takahashi M. Plasma amino acid analysis for diagnosis and amino acid-based metabolic networks. Curr Opin Clin Nutr Metab Care.

9. Kurman RJ, Shih I-M. The Origin and Pathogenesis of Epithelial Ovarian Cancer: A Proposed Unifying Theory. Am J Surg Pathol. 2010 Mar;34(3):433-43.

10. Filee R, Schoos R, Boemer F. Evaluation of physiological amino acids profiling by tandem mass spectrometry. JIMD Rep. Springer; 2014;13:119-28.

11. Tsun Z-Y, Possemato R. Amino acid management in cancer. Semin Cell Dev Biol. NIH Public Access; 2015 Jul;43:22-32.

12. Kubota A, Meguid MM, Hitch DC. Amino acid profiles correlate diagnostically with organ site in three kinds of malignant tumors. Cancer. Wiley Subscription Services, Inc., A Wiley Company; 1992 May 1;69(9):2343-8.

13. Leichtle AB, Nuoffer J-M, Ceglarek U, Kase J, Conrad T, Witzigmann H, et al. Serum amino acid profiles and their alterations in colorectal cancer. Metabolomics. Springer US; 2012 Aug 16;8(4):643-53.

14. Ihata Y, Miyagi E, Numazaki R, Muramatsu T, Imaizumi A, Yamamoto H, et al. Amino acid profile index for early detection of endometrial cancer: verification as a novel diagnostic marker. Int J Clin Oncol. 2014 Apr 23;19(2):364-72. 
15. Zhou M, Guan W, Walker LD, Mezencev R, Benigno BB, Gray A, et al. Rapid Mass Spectrometric Metabolic Profiling of Blood Sera Detects Ovarian Cancer with High Accuracy. Cancer Epidemiol Biomarkers Prev. 2010 Sep 1;19(9):2262-71.

16. Hilvo M, de Santiago I, Gopalacharyulu P, Schmitt WD, Budczies J, Kuhberg M, et al. Accumulated Metabolites of Hydroxybutyric Acid Serve as Diagnostic and Prognostic Biomarkers of Ovarian High-Grade Serous Carcinomas. Cancer Res. 2016 Feb 15;76(4):796-804.

17. Garcia E, Andrews C, Hua J, Kim HL, Sukumaran DK, Szyperski T, et al. Diagnosis of Early Stage Ovarian Cancer by ${ }^{1}$ H NMR Metabonomics of Serum Explored by Use of a Microflow NMR Probe. J Proteome Res. 2011 Apr 1;10(4):1765-71.

18. Bachmayr-Heyda A, Aust S, Auer K, Meier SM, Schmetterer KG, Dekan S, et al. Integrative Systemic and Local Metabolomics with Impact on Survival in High-Grade Serous Ovarian Cancer. Clin Cancer Res. 2017;

19. Buas MF, Gu H, Djukovic D, Zhu J, Drescher CW, Urban N, et al. Identification of novel candidate plasma metabolite biomarkers for distinguishing serous ovarian carcinoma and benign serous ovarian tumors. Gynecol Oncol. 2016 Jan;140(1):138-44.

20. Ke C, Hou Y, Zhang H, Fan L, Ge T, Guo B, et al. Large-scale profiling of metabolic dysregulation in ovarian cancer. Int J Cancer. 2014 Jun 1;136(3):n/a-n/a.

21. Miyagi E, Maruyama Y, Mogami T, Numazaki R, Ikeda A, Yamamoto H, et al. Comparison of plasma amino acid profile-based index and CA125 in the diagnosis of epithelial ovarian cancers and borderline malignant tumors. Int J Clin Oncol. 2017 Feb $13 ; 22(1): 118-25$.

22. Zhang T, Wu X, Yin M, Fan L, Zhang H, Zhao F, et al. Discrimination between malignant and benign ovarian tumors by plasma metabolomic profiling using ultra 
performance liquid chromatography/mass spectrometry. Clin Chim Acta. 2012 May 18;413(9-10):861-8.

23. Pavlova NN, Thompson CB. The Emerging Hallmarks of Cancer Metabolism. Cell Metab. 2016 Jan 12;23(1):27-47.

24. Medina VA, Rivera ES. Histamine receptors and cancer pharmacology. Br J Pharmacol. Wiley-Blackwell; 2010 Oct;161(4):755-67.

25. Uhlen M, Fagerberg L, Hallstrom BM, Lindskog C, Oksvold P, Mardinoglu A, et al. Tissue-based map of the human proteome. Science (80- ). 2015 Jan 23;347(6220):1260419-1260419.

26. Zheng Y, Ceglarek U, Huang T, Li L, Rood J, Ryan DH, et al. Weight-loss diets and 2y changes in circulating amino acids in 2 randomized intervention trials. Am J Clin Nutr. 2016 Feb 1;103(2):505-11.

27. Koshiyama M, Matsumura N, Konishi I. Recent concepts of ovarian carcinogenesis: type I and type II. Biomed Res Int. Hindawi Publishing Corporation; 2014;2014:934261.

28. Matysiak J, Dereziński P, Klupczyńska A, Matysiak J, Kaczmarek E, Kokot ZJ. Effects of a honeybee sting on the serum free amino acid profile in humans. PLoS One. Public Library of Science; 2014;9(7):e103533.

29. Xia J, Wishart DS. Using MetaboAnalyst 3.0 for Comprehensive Metabolomics Data Analysis. In: Current Protocols in Bioinformatics. Hoboken, NJ, USA: John Wiley \& Sons, Inc.; 2016. p. 14.10.1-14.10.91. 


\section{Supplementary Files}

This is a list of supplementary files associated with this preprint. Click to download.

- SFAAprofilinginovatumorsJOVRTable2.docx

- SFAAprofilinginovatumorsJOVRTable4.docx

- SFAAprofilinginovatumorsJOVRTableS1.docx 